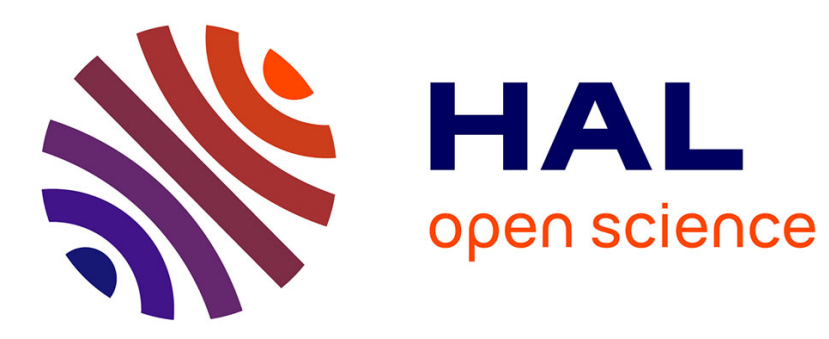

\title{
Guidance of an ultrasound probe by visual servoing.
}

A. Krupa, François Chaumette

\section{To cite this version:}

A. Krupa, François Chaumette. Guidance of an ultrasound probe by visual servoing.. Advanced Robotics, 2006, 20 (11), pp.1203-1218. inria-00350296

\section{HAL Id: inria-00350296 \\ https://hal.inria.fr/inria-00350296}

Submitted on 6 Jan 2009

HAL is a multi-disciplinary open access archive for the deposit and dissemination of scientific research documents, whether they are published or not. The documents may come from teaching and research institutions in France or abroad, or from public or private research centers.
L'archive ouverte pluridisciplinaire HAL, est destinée au dépôt et à la diffusion de documents scientifiques de niveau recherche, publiés ou non, émanant des établissements d'enseignement et de recherche français ou étrangers, des laboratoires publics ou privés. 


\title{
Full paper
}

\section{Guidance of an ultrasound probe by visual servoing}

\author{
ALEXANDRE KRUPA* and FRANÇOIS CHAUMETTE \\ IRISA - INRIA Rennes, Campus de Beaulieu, 35042 Rennes Cedex, France
}

Received 29 November 2005; accepted 20 January 2006

\begin{abstract}
A new visual servoing technique based on two-dimensional (2-D) ultrasound (US) image is proposed in order to control the motion of an US probe held by a medical robot. In opposition to a standard camera which provides a projection of the three-dimensional (3-D) scene to a 2-D image, US information is strictly in the observation plane of the probe and consequently visual servoing techniques have to be adapted. In this paper the coupling between the US probe and a motionless crossed string phantom used for probe calibration is modeled. Then a robotic task is developed which consists of positioning the US image on the intersection point of the crossed string phantom while moving the probe to different orientations. The goal of this task is to optimize the procedure of spatial parameter calibration of 3-D US systems.
\end{abstract}

Keywords: Ultrasound probe guidance; visual servoing; calibration; medical robotics; redundancy.

\section{INTRODUCTION}

Among the numerous medical imaging modalities in use today, ultrasound (US) systems are currently the most commonly employed due to their ease of use and minimal amount of harmful side-effects. Three-dimensional (3-D) spatial US imaging is usually chosen for clinical applications such as cardiology, obstetrics and vascular imaging. For the past few years, 3-D US sensors have been available for this kind of imagery, but they currently provide only low voxel resolution and, because of their high cost, they are not as prevalent in clinics as conventional two-dimensional (2-D) US systems. Nevertheless, an alternative technique called '3-D free-hand US imaging' [1] consists of measuring the relative displacement between each image captured by a 2-D US system in order to position it in a 3-D reference frame $\mathcal{F}_{0}$ to obtain the volume information as illustrated in Fig. 1. Usually the localization system, which can be magnetic, optic, acoustic or mechanical,

\footnotetext{
*To whom correspondence should be addressed. E-mail: alexandre.krupa@ irisa.fr
} 


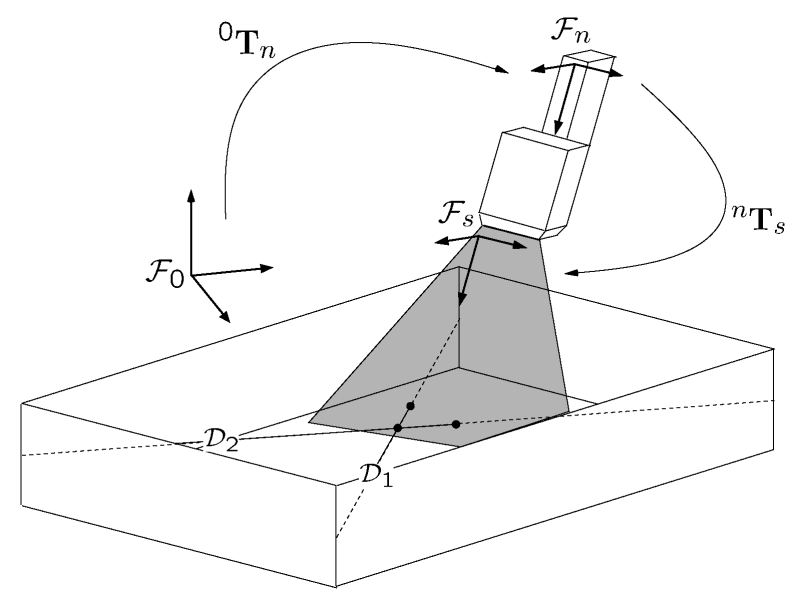

Figure 1. 3-D US imaging with a 2-D probe and spatial calibration procedure by using a crossed string phantom.

is fixed to the US probe (reference frame $\mathcal{F}_{\mathrm{n}}$ ) and continuously gives its position and orientation defined by the homogeneous transformation ${ }^{0} \mathbf{T}_{\mathrm{n}}$. In order to obtain a good accuracy of the 3-D reconstruction, it is crucial that the localization system provides a very low position error and that the spatial calibration parameters of the US system are known at best. These spatial parameters include the rigid transformation ${ }^{n} \mathbf{T}_{\mathrm{s}}$ from the position sensor to the image frame, and the US image scaling factors $S_{x}$ and $S_{y}$. In the literature, several methods have been proposed to identify the spatial calibration parameters of 3-D free-hand US systems. The principle of these methods is to capture a set of US images of a known object immersed in water for different measured positions of the probe and then to off-line estimate the spatial parameters by coupling visual features extracted from each US image to the geometrical properties of the object. For example, in Ref. [2], a method is presented whereby the intersection point $P^{*}$ (see Fig. 1) of a fixed, crossed string phantom constituted by two converging straight lines $\mathcal{D}_{1}$ and $\mathcal{D}_{2}$, immersed in water has to be positioned in the US image for different orientations of the US probe; in Ref. [3], another method is developed using a plane phantom.

Our research aim is to develop a robotic system that will optimize 3-D US imaging by automatically moving the US probe during a medical examination. Unlike Refs [4-6] where teleoperated master/slave systems are presented, we plan to control the robot directly from visual information extracted from US images. The idea is to perform automatically by visual servoing the 3-D acquisition of a volume specified by the clinician. This will allow the clinician to repeat the examination of a patient on different dates in order to observe quantitatively the pathology evolution under the same conditions. Toward that end, a robotic system [7] has already been developed to automatically perform the 3-D US acquisition of cardiovascular 
pathologies. However, this system does not use the US visual information and requires the clinician to manually set the input and output ports of the trajectory. Up until now, only a few studies have been made on visual servoing using information from 2-D US images. In Ref. [8], visual servoing is used to center within the 2-D US image a point corresponding to the section center of an artery during the probe displacement along a one-dimensional trajectory. Of the 6 d.o.f. available to the robot holding the US probe, only 3 d.o.f. in the US observation plane are controlled by visual servoing, while the other 3 d.o.f. are teleoperated by the user. In another work [9], the authors present a robotic system for needle insertion with the US probe rigidly fixed to the base of a small 2-d.o.f. robot held by a passive 5-d.o.f. mechanical architecture. The probe is positioned in such a way that the needle is always visible in the US image as a straight line. Once again, only the d.o.f. (here 2) in the US observation plane are controlled by visual servoing. More recently, a study has been presented where 4 d.o.f., which are not necessary in the observation plane of the probe, are controlled by visual servoing [10]. The goal of this last work is to automatically move a laparoscopic instrument to a desired position indicated by a surgeon in the US image which is provided by a motionless probe.

In this paper we present the first results of our research concerning the optimization of 3-D US imaging by the use of a robotic system. In order to facilitate the spatial parameters calibration of the 3-D US system, we propose to develop a robotic task that consists of automatically positioning the US image plane on the intersection point of the crossed string phantom used by the Detmer calibration method [2], while moving the probe to different orientations. This task will be performed by controlling the 6 d.o.f. of the manipulator holding the US probe.

This paper is composed as follows. In Section 2, we model the coupling between the observation plane of the probe and the two straight lines describing the motionless crossed string phantom. In Section 3, the robotic task is formulated, the visual features are defined and the visual servoing control law is developed. The redundancy formalism [11] is applied in order to move the probe to different orientations. Then, Section 4 presents simulation results of the proposed control law and is followed by the conclusion.

\section{MODELING}

\subsection{Geometrical modeling}

Let $\mathcal{F}_{0}, \mathcal{F}_{\mathrm{s}}$ and $\mathcal{F}_{\mathrm{n}}$ be, respectively, the frames of reference attached to the robot base, the US probe and the robot end-effector as illustrated in Fig. 2. The observation plane $\mathcal{P}_{\pi}$ of the ultrasound probe is defined by the $\vec{u}_{x}$ and $\vec{u}_{y}$ axes of $\mathcal{F}_{\mathrm{s}}$. Let $P$ be the intersection point between a straight line $\mathcal{D}$ (not collinear to $\mathcal{P}_{\pi}$ ) and 


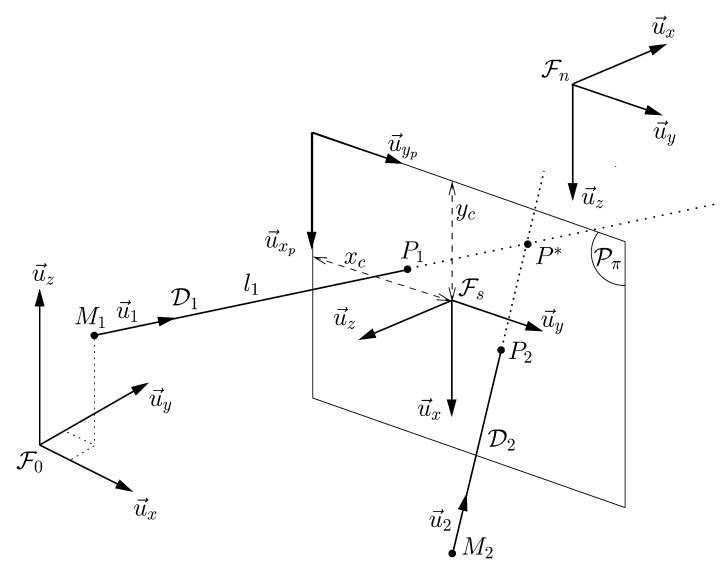

Figure 2. US probe coupling with two converging straight lines.

the observation plane $\mathcal{P}_{\pi}$. The coordinates of $P$ expressed in the robot base frame $\mathcal{F}_{0}$ are given by:

$$
{ }^{0} \mathbf{P}={ }^{0} \mathbf{M}+l{ }^{0} \mathbf{u},
$$

where ${ }^{0} \mathbf{M}$ are the coordinates of a point $M$ which belongs to $\mathcal{D},{ }^{0} \mathbf{u}$ is the unitary vector of $\mathcal{D}$, and $l$ is the distance between $M$ and $P$ (the left subscript zero denotes that the components are expressed in $\mathcal{F}_{0}$ ). By expressing $P$ in the probe frame $\mathcal{F}_{\mathrm{s}}$ we obtain:

$$
{ }^{\mathrm{s}} \mathbf{P}={ }^{\mathrm{s}} \mathbf{t}_{0}+{ }^{\mathrm{s}} \mathbf{R}_{0}\left({ }^{0} \mathbf{M}+l{ }^{0} \mathbf{u}\right) .
$$

Here the vector ${ }^{\mathrm{s}} \mathbf{t}_{0}$ and the matrix ${ }^{\mathrm{s}} \mathbf{R}_{0}$ represent, respectively, the translation and the rotation from the probe frame to the base frame. As $P$ belongs to $\mathcal{P}_{\pi}$, its projection on the $\vec{u}_{z}$ axis of $\mathcal{F}_{\mathrm{s}}$ is null. This projection expressed in $\mathcal{F}_{\mathrm{s}}$ gives:

$$
{ }^{\mathrm{s}} \mathbf{u}_{z}^{\top \mathrm{s}} \mathbf{P}=0
$$

with ${ }^{\mathrm{s}} \mathbf{u}_{z}=(0,0,1)$. It follows that ${ }^{\mathrm{s}} \mathbf{P}=(x, y, z=0)$. The distance $l$ can then be obtained by substituting (2) in (3):

$$
l=-\frac{{ }^{\mathrm{s}} \mathbf{u}_{z}^{\top}\left({ }^{\mathrm{s}} \mathbf{t}_{0}+{ }^{\mathrm{s}} \mathbf{R}_{0}{ }^{0} \mathbf{M}\right)}{{ }^{\mathrm{s}} \mathbf{u}_{z}^{\top}{ }^{\mathrm{s}} \mathbf{R}_{0}{ }^{0} \mathbf{u}} .
$$

From (2) and (4) the coordinates of $P$ expressed in the probe frame can then be computed if the geometrical parameters ${ }^{\mathrm{s}} \mathbf{t}_{0},{ }^{\mathrm{s}} \mathbf{R}_{0},{ }^{0} \mathbf{M}$ and ${ }^{0} \mathbf{u}$ are known.

\subsection{Interaction matrix of a point belonging to the observation plane and a straight line}

In classical visual servoing, the interaction matrix $\mathbf{L}_{\mathbf{s}}$ is used to link the variation of the visual information $\mathbf{s}$ to the relative kinematic screw $\mathbf{v}$ between the camera and 
the scene:

$$
\dot{\mathbf{s}}=\mathbf{L}_{\mathbf{s}} \mathbf{v}
$$

In our system, the visual information associated to the point $P$ are its 2-D coordinates $\mathbf{p}=(x, y)$ expressed in the US probe frame. Since the image coordinates are measured in pixels in the 2-D image frame $\left\{\vec{u}_{x_{\mathrm{p}}}, \vec{u}_{y_{\mathrm{p}}}\right\}$ attached at the left-top corner of the image plane, the following variable transformation has to be made to obtain $\mathbf{p}=(x, y)$ which is expressed in $\mathrm{m}$ :

$$
x=\left(x_{\mathrm{p}}-x_{\mathrm{c}}\right) S_{x} \quad \text { and } \quad y=\left(y_{\mathrm{p}}-y_{\mathrm{c}}\right) S_{y},
$$

where $\left(x_{\mathrm{p}}, y_{\mathrm{p}}\right)$ are the pixel coordinates of $P,\left(x_{\mathrm{c}}, y_{\mathrm{c}}\right)$ are the pixel coordinates of the image center, and $S_{x}$ and $S_{y}$ are, respectively, the height and width of a pixel. The analytical form of the interaction matrix related to $\mathbf{p}$ is determined by calculating the time derivative of (2):

$$
{ }^{\mathrm{s}} \dot{\mathbf{P}}={ }^{\mathrm{s}} \dot{\mathbf{t}}_{0}+{ }^{\mathrm{s}} \dot{\mathbf{R}}_{0}\left({ }^{0} \mathbf{M}+l{ }^{0} \mathbf{u}\right)+{ }^{\mathrm{s}} \mathbf{R}_{0}\left({ }^{0} \dot{\mathbf{M}}+i^{0} \mathbf{u}\right) .
$$

As the point $M$ is fixed with respect to $\mathcal{F}_{0}$, we have:

$$
{ }^{\mathrm{s}} \dot{\mathbf{P}}={ }^{\mathrm{s}} \dot{\mathbf{t}}_{0}+{ }^{\mathrm{s}} \dot{\mathbf{R}}_{0}\left({ }^{0} \mathbf{M}+l{ }^{0} \mathbf{u}\right)+{ }^{\mathrm{s}} \mathbf{R}_{0} i^{0} \mathbf{u}
$$

Let us define $\mathbf{v}=(\boldsymbol{v}, \boldsymbol{\omega})$ as the velocity screw of the probe expressed in $\mathcal{F}_{\mathrm{s}}$ with $\boldsymbol{v}=\left(v_{x}, v_{y}, v_{z}\right)$ the translational velocity vector and $\boldsymbol{\omega}=\left(\omega_{x}, \omega_{y}, \omega_{z}\right)$ the angular velocity vector of the probe frame with respect to the base frame. The time derivative of the rotational matrix is linked to $\omega$ by [11]:

$$
{ }^{\mathrm{s}} \dot{\mathbf{R}}_{0}=-[\omega] \times{ }^{\mathrm{s}} \mathbf{R}_{0}
$$

with $[\omega]_{\times}$being the skew symmetric matrix associated with the angular velocity vector $\omega$. The term ${ }^{\mathrm{s}} \dot{\mathbf{t}}_{0}$ is the velocity of the base frame origin with respect to the probe frame. It is related to the velocity screw $\mathbf{v}$ of the probe by the following fundamental equation of kinematics:

$$
{ }^{\mathrm{s}} \dot{\mathbf{t}}_{0}=-\boldsymbol{v}+\left[{ }^{\mathrm{s}} \mathbf{t}_{0}\right]_{\times} \boldsymbol{\omega}=\left[-\mathbf{I}_{3} \quad\left[{ }^{\mathrm{s}} \mathbf{t}_{0}\right]_{\times}\right] \mathbf{v},
$$

where $\left[{ }^{\mathrm{s}} \mathbf{t}_{0}\right]_{\times}$is the skew symmetric matrix associated with ${ }^{\mathrm{s}} \mathbf{t}_{0}$. By substituting (2), (9) and (10) in (8), we obtain:

$$
{ }^{\mathrm{s}} \dot{\mathbf{P}}=\left[{ }^{\mathrm{s}} \mathbf{P}\right]_{\times} \boldsymbol{\omega}+i^{\mathrm{s}} \mathbf{R}_{0}{ }^{0} \mathbf{u}-\boldsymbol{v} .
$$

By applying the projection relation ${ }^{\mathrm{s}} \mathbf{u}_{z}^{\top \mathrm{s}} \dot{\mathbf{P}}=0$, the time derivative of the distance $l$ can then be extracted:

$$
i=\frac{{ }^{\mathrm{s}} \mathbf{u}_{z}^{\top} \boldsymbol{v}-{ }^{\mathrm{s}} \mathbf{u}_{z}^{\top}\left[{ }^{\mathrm{s}} \mathbf{P}\right]_{\times} \boldsymbol{\omega}}{{ }^{\mathrm{s}} \mathbf{u}_{z}^{\top \mathrm{s}} \mathbf{R}_{0}{ }^{0} \mathbf{u}},
$$


which after substitution in (11) gives the following expression:

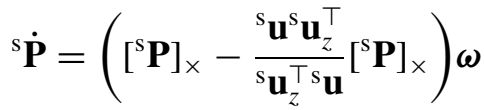

$$
\begin{aligned}
& +\left(\frac{{ }^{\mathrm{s}} \mathbf{u}^{\mathrm{s}} \mathbf{u}_{z}^{\top}}{{ }^{\mathrm{s}} \mathbf{u}_{z}^{\top \mathrm{s}} \mathbf{u}}-\mathbf{I}_{3}\right) \boldsymbol{v},
\end{aligned}
$$

where ${ }^{\mathrm{s}} \mathbf{u}={ }^{\mathrm{s}} \mathbf{R}_{0}{ }^{0} \mathbf{u}=\left(u_{x}, u_{y}, u_{z}\right)$ is the unitary vector of $\mathcal{D}$ expressed in the probe frame. Finally, we can determine the interaction matrix $\mathbf{L}_{\mathbf{p}}$ related to $\mathbf{p}$ by developing the two first rows of (13):

$$
\dot{\mathbf{p}}=(\dot{x}, \dot{y})=\mathbf{L}_{\mathbf{p}} \mathbf{v}
$$

with

$$
\mathbf{L}_{\mathbf{p}}=\left[\begin{array}{cccccc}
-1 & 0 & \frac{u_{x}}{u_{z}} & \frac{u_{x}}{u_{z}} y & -\frac{u_{x}}{u_{z}} x & y \\
0 & -1 & \frac{u_{y}}{u_{z}} & \frac{u_{y}}{u_{z}} y & -\frac{u_{y}}{u_{z}} x & -x
\end{array}\right]
$$

This matrix depends on the components of the unitary vector ${ }^{\mathrm{s}} \mathbf{u}$ of the straight line $\mathcal{D}$ and the 2-D coordinates $\mathbf{p}$, all expressed in the probe frame. The condition to compute $\mathbf{L}_{\mathrm{p}}$ is that $u_{z} \neq 0$. This is verified when $\mathcal{D}$ is not collinear to the observation plan $\mathcal{P}_{\pi}$. Note that if $P$ coincides with the origin of $\mathcal{F}_{\mathrm{s}}$ then $\mathbf{p}$ is invariant to the rotational motion of the US probe and if $\mathcal{D}$ is orthogonal to $\mathcal{P}_{\pi}\left(u_{x}=0\right.$ and $\left.u_{y}=0\right)$ then $\mathbf{p}$ is invariant to the translational motion along $\vec{u}_{z}$.

\section{VISUAL SERVOING}

First, let us formulate the robotic task to achieve. The goal is to position the intersection point $P^{*}$ between two converging straight lines $\mathcal{D}_{1}$ and $\mathcal{D}_{2}$ not collinear to the US observation plan $\mathcal{P}_{\pi}$ (see Fig. 2) on a target point defined in the US image with a set of different orientations of the probe. For each orientation, the endeffector pose will be recorded once the point is positioned in the image in order to estimate the spatial calibration parameters of the US system by the use of the offline Detmer method [2]. The visual task consists in centering the points $P_{1}$ and $P_{2}$ on a target indicated in the image.

\subsection{Vision-based task function}

The visual features $\mathbf{s}$ we chose are the 2 -D coordinates $\mathbf{p}_{1}=\left(x_{1}, y_{1}\right)$ and $\mathbf{p}_{2}=$ $\left(x_{2}, y_{2}\right)$ of points $P_{1}$ and $P_{2}$ expressed in the probe frame:

$$
\mathbf{s}=\left(x_{1}, y_{1}, x_{2}, y_{2}\right)
$$


The interaction matrix related to $\mathbf{s}$ is obtained by stacking the two interaction matrices associated to $\mathbf{p}_{1}$ and $\mathbf{p}_{2}$ and whose form is given by (15):

$$
\mathbf{L}_{\mathbf{s}}=\left[\begin{array}{cccccc}
-1 & 0 & \frac{u_{1_{x}}}{u_{1_{z}}} & \frac{u_{1_{x}}}{u_{1_{z}}} y_{1} & -\frac{u_{1_{x}}}{u_{1_{z}}} x_{1} & y_{1} \\
0 & -1 & \frac{u_{1_{y}}}{u_{1_{z}}} & \frac{u_{1_{y}}}{u_{1_{z}}} y_{1} & -\frac{u_{y_{1}}}{u_{1_{z}}} x_{1} & -x_{1} \\
-1 & 0 & \frac{u_{2_{x}}}{u_{2_{z}}} & \frac{u_{2_{x}}}{u_{2_{z}}} y_{2} & -\frac{u_{2_{x}}}{u_{2_{z}}} x_{2} & y_{2} \\
0 & -1 & \frac{u_{2_{y}}}{u_{2_{z}}} & \frac{u_{2_{y}}}{u_{2 z}} y_{2} & -\frac{u_{y_{2}}}{u_{2 z}} x_{2} & -x_{2}
\end{array}\right] .
$$

Note that the rank of $\mathbf{L}_{\mathbf{s}}$ is 4 except when the two points are joined. In this last case the rank is reduced to 3 . We will see in the Section 4 how to cope with the rank change. The visual servoing task can be expressed as a regulation to zero of the visual error:

$$
\mathbf{e}_{1}(\mathbf{r}(t))=\mathbf{s}(\mathbf{r}(t))-\mathbf{s}^{*},
$$

where $\mathbf{s}^{*}$ is the reference value of the visual features to be reached and $\mathbf{s}$ is the value of the visual features currently observed by the US probe. The features depend on the relative position $\mathbf{r}$ between the probe and the scene.

The robot holding the US probe has $n=6$ d.o.f. and the dimension of the visionbased task $\mathbf{e}_{1}$ is at most 4 . This means that the vision-based task does not constrain all the robot's 6 d.o.f. Consequently, it is possible to use the other d.o.f. to perform a secondary task such as the changes of orientation of the probe. Note that if three straight lines are used, we have generally $m=n=6$ and then the 6 d.o.f. are controlled by the vision task.

\subsection{Redundancy formalism}

Here, we present the redundancy formalism [11]. It has first been used for visual servoing in Ref. [12] and in numerous applications since (e.g., avoiding visual features occlusion [13] or human-machine cooperation using vision control [14]). The idea is to use the d.o.f. left by a main task $\mathbf{e}_{1}$ of dimension $m<n$, to realize a secondary task $\mathbf{g}^{\top}=\frac{\partial h}{\partial \mathbf{r}}$ at best without disturbing the first one. Generally, the realization of a secondary goal is expressed as a minimization of a cost function $h$ under the constraint that the main task is achieved, i.e., $\mathbf{e}_{1}(\mathbf{r}(t))=0$. The determination of d.o.f. which are left by the main task requires the computation of the null space of the interaction matrix $\mathbf{L}_{\mathbf{e}_{1}}$ of the task $\mathbf{e}_{1}$. In our case, we have of course $\mathbf{L}_{\mathbf{e}_{1}}=\mathbf{L}_{\mathbf{s}}$. The global task function is given by [11]:

$$
\mathbf{e}=\hat{\mathbf{L}}_{\mathbf{s}}^{+} \mathbf{e}_{1}+\left(\mathbf{I}_{\mathrm{n}}-\hat{\mathbf{L}}_{\mathbf{s}}^{+} \hat{\mathbf{L}}_{\mathbf{s}}\right) \mathbf{g}^{\top}
$$


where $\hat{\mathbf{L}}_{\mathbf{s}}^{+}$is the pseudo-inverse of an estimation $\hat{\mathbf{L}}_{\mathbf{s}}$ of the interaction matrix and $\left(\mathbf{I}_{\mathrm{n}}-\hat{\mathbf{L}}_{\mathbf{s}}^{+} \hat{\mathbf{L}}_{\mathbf{s}}\right)$ is an orthogonal projector operator which projects $\mathbf{g}^{\top}$ in the null space of $\hat{\mathbf{L}}_{\mathbf{s}}$ in order that the second task does not disturb the first one.

\subsection{Control law}

Usually, the control law is obtained by trying to make the global task exponentially decrease in order to behave like a first-order decoupled system. If the observed object is static (which is our case because the crossed string phantom is motionless), this is achieved by applying the following control screw velocity to the probe [12]:

$$
\mathbf{v}=-\lambda \mathbf{e}-\left(\mathbf{I}_{\mathrm{n}}-\hat{\mathbf{L}}_{\mathbf{s}}^{+} \hat{\mathbf{L}}_{\mathbf{s}}\right) \frac{\partial \mathbf{g}^{\top}}{\partial t},
$$

where $\lambda$ is the proportional coefficient involved in the exponential convergence of $\mathbf{e}$ and $\hat{\mathbf{L}}_{\mathbf{s}}$ is an approximation of the interaction matrix. An on-line estimation of $\mathbf{L}_{\mathbf{s}}$ is presented in Section 3.5.

In practice, we consider the input of the robot controller as the kinematic screw $\mathbf{v}_{\mathrm{n}}$ of the end-effector. It is linked to the kinematic screw $\mathbf{v}$ of the US probe by:

$$
\mathbf{v}_{\mathrm{n}}=\left(\begin{array}{cc}
{ }^{\mathrm{n}} \mathbf{R}_{\mathrm{s}} & {\left[{ }^{\mathrm{n}} \mathbf{t}_{\mathrm{s}}\right]_{\times}{ }^{\mathrm{n}} \mathbf{R}_{\mathrm{s}}} \\
\mathbf{0}_{3} & { }^{\mathrm{n}} \mathbf{R}_{\mathrm{s}}
\end{array}\right) \mathbf{v},
$$

where ${ }^{\mathrm{n}} \mathbf{t}_{\mathrm{s}}$ and ${ }^{\mathrm{n}} \mathbf{R}_{\mathrm{s}}$ are the translation vector and the rotation matrix from the endeffector frame to the probe frame. These two parameters with the image scaling factors $S_{x}$ and $S_{y}$ correspond to the spatial parameters of the US system. Since these parameters are not perfectly known before using the off-line Detmer calibration method, we set them to rough values. We will see in Section 4 that this will not affect the task performance due to the well-known robustness property of imagebased visual servoing.

\subsection{Application of the redundancy formalism to our robotic task}

We define our secondary task as the minimization of the rotation ${ }^{\mathrm{s}} \mathbf{R}_{\mathrm{s}^{*}}$ from the current orientation ${ }^{0} \mathbf{R}_{\mathrm{s}}$ of the probe to a desired orientation ${ }^{0} \mathbf{R}_{\mathrm{s}^{*}}$ expressed in the robot base frame (with ${ }^{\mathrm{s}} \mathbf{R}_{\mathrm{s}^{*}}={ }^{0} \mathbf{R}_{\mathrm{s}}^{\top 0} \mathbf{R}_{\mathrm{s}^{*}}$ ). To describe the rotation ${ }^{\mathrm{s}} \mathbf{R}_{\mathrm{s}^{*}}$, we chose the minimal representation $\theta \mathbf{u}$ where $\theta$ represents the angle around the unitary rotation axis $\mathbf{u}$. The representation $\theta \mathbf{u}$ is obtained from the coefficients $r_{i j(i=1 . .3, j=1 . .3)}$ of the rotation matrix ${ }^{\mathrm{s}} \mathbf{R}_{\mathrm{s}^{*}}$ by the following equation:

$$
\theta \mathbf{u}=\frac{1}{2 \operatorname{sinc}(\theta)}\left(\begin{array}{l}
r_{32}-r_{23} \\
r_{13}-r_{31} \\
r_{21}-r_{12}
\end{array}\right)
$$

where $\theta=\arccos \left(\left(r_{11}+r_{22}+r_{33}-1\right) / 2\right)$ and $\operatorname{sinc}(\theta)=\sin (\theta) / \theta$ is the sinus cardinal function. The secondary task consists to minimize $\theta \mathbf{u}$ or at best to regulate 
it towards zero if the d.o.f. left free by the main task make it possible. To take into account the secondary task, we define the following quadratic cost function:

$$
h=\frac{1}{2} \theta \mathbf{u}^{\top} \theta \mathbf{u},
$$

and by computing the gradient of $h$ and its partial time derivative, we get:

$$
\mathbf{g}=\left[\mathbf{0}_{[1 \times 3]} \theta \mathbf{u}^{\top}\right] \text { and } \frac{\partial \mathbf{g}}{\partial t}=0 .
$$

\subsection{On-line estimation of the interaction matrix}

The interaction matrix $\mathbf{L}_{\mathbf{s}}$ depends on the unitary vectors ${ }^{\mathrm{s}} \mathbf{u}_{1}$ and ${ }^{\mathrm{s}} \mathbf{u}_{2}$ of straight lines $\mathcal{D}_{1}$ and $\mathcal{D}_{2}$, and the 2-D coordinates $\mathbf{p}_{1}, \mathbf{p}_{2}$ of points $P_{1}$ and $P_{2}$ all expressed in the probe frame. In practice $\mathbf{p}_{1}, \mathbf{p}_{2}$ are provided from their pixel coordinates measured in the US image (see (6)). Nevertheless ${ }^{5} \mathbf{u}_{1}$ and ${ }^{\mathrm{s}} \mathbf{u}_{2}$ are not known, and we have to on-line estimate them. To do this, we use a recursive least-squares algorithm delivered below. For the sake of simplicity, in the sequel we give the method for one straight line $\mathcal{D}$. First, let us define a frame $\mathcal{F}_{\mathrm{f}}$ fixed to the scene wherein projection ${ }^{\mathrm{f}} \mathbf{u}$ of ${ }^{\mathrm{s}} \mathbf{u}$ is constant and the following minimal representations of $\mathcal{D}$ can be used:

$$
x=a z+c \quad \text { and } \quad y=b z+d,
$$

where $x, y, z$ are the coordinates expressed in $\mathcal{F}_{\mathrm{f}}$ of any point belonging to the straight line $\mathcal{D}$ and $a, b, c, d$ are constant parameters. This minimal representation is always valid if $\mathcal{D}$ is not collinear to the plane described by $\vec{u}_{x}$ and $\vec{u}_{y}$ axes of $\mathcal{F}_{\mathrm{f}}$. To assure this, a good choice for $\mathcal{F}_{\mathrm{f}}$ is the probe frame frozen at the beginning of the servoing $\mathcal{F}_{\mathrm{f}}=\mathcal{F}_{\mathrm{S}}(t=0)$. By rewriting (25) in a vectorial system form we have:

$$
\mathbf{Y}=(x, y)=\boldsymbol{\Phi}^{\top} \boldsymbol{\theta},
$$

with $\boldsymbol{\theta}=(a, b, c, d)$ the parameter vector to estimate and:

$$
\boldsymbol{\Phi}^{\top}=\left(\begin{array}{cccc}
z & 0 & 1 & 0 \\
0 & z & 0 & 1
\end{array}\right) .
$$

This system can be solved if we have at least the coordinates measurement of two different points belonging to $\mathcal{D}$. Of course the more points we have, the better will be the estimation. In our approach we take into account all coordinates ${ }^{\mathrm{f}} \mathbf{P}_{[k]}$ of $P$ measured at each iteration $k$ during the servoing and expressed in $\mathcal{F}_{\mathrm{f}}$. In discrete time, the least-squares method consists in computing the estimation value $\hat{\boldsymbol{\theta}}_{[k]}$ that minimizes the following quadratic sum of the modeling error [15]:

$$
J\left(\hat{\boldsymbol{\theta}}_{[k]}\right)=\sum_{i=0}^{k}\left(\mathbf{Y}_{[i]}-\boldsymbol{\Phi}_{[i]}^{\top} \hat{\boldsymbol{\theta}}_{[k]}\right)^{\top}\left(\mathbf{Y}_{[i]}-\boldsymbol{\Phi}_{[i]}^{\top} \hat{\boldsymbol{\theta}}_{[k]}\right) .
$$


Therefore, $\hat{\boldsymbol{\theta}}_{[k]}$ is obtained by nullifying the gradient of $J\left(\hat{\boldsymbol{\theta}}_{[k]}\right)$ which is given by:

$$
\nabla J\left(\hat{\boldsymbol{\theta}}_{[k]}\right)=-2 \sum_{i=0}^{k} \boldsymbol{\Phi}_{[i]}\left(\mathbf{Y}_{[i]}-\boldsymbol{\Phi}_{[i]}^{\top} \hat{\boldsymbol{\theta}}_{[k]}\right)=0 .
$$

Finally, we obtain the following recursive expression:

$$
\hat{\boldsymbol{\theta}}_{[k]}=\hat{\boldsymbol{\theta}}_{[k-1]}+\mathbf{F}_{[k]} \boldsymbol{\Phi}_{[k]}\left(\mathbf{Y}_{[k]}-\boldsymbol{\Phi}_{[k]}^{\top} \hat{\boldsymbol{\theta}}_{[k-1]}\right),
$$

where $\mathbf{F}_{[k]}$ is a covariance matrix such that $\mathbf{F}_{[k]}=\mathbf{F}_{[k]}^{\top}>0$ and whose recursive expression is:

$$
\mathbf{F}_{[k]}^{-1}=\mathbf{F}_{[k-1]}^{-1}+\boldsymbol{\Phi}_{[k]} \boldsymbol{\Phi}_{[k]}^{\top} .
$$

In practice we set initial values $\mathbf{F}_{[0]}=f_{0} \mathbf{I}_{4}$ with $f_{0}>0$ and $\hat{\boldsymbol{\theta}}_{[0]}=\boldsymbol{\theta}_{0}$. Once $\hat{\boldsymbol{\theta}}=(\hat{a}, \hat{b}, \hat{c}, \hat{d})$ is computed, the estimated unitary vector ${ }^{\mathrm{f}} \hat{\mathbf{u}}$ of $\mathcal{D}$ is linked to parameters $\hat{a}$ and $\hat{b}$ by:

$$
{ }^{\mathrm{f}} \hat{\mathbf{u}}=(\hat{a}, \hat{b}, 1) /\|(\hat{a}, \hat{b}, 1)\|,
$$

and expressed in the probe frame with:

$$
{ }^{\mathrm{s}} \hat{\mathbf{u}}={ }^{\mathrm{s}} \mathbf{R}_{0}{ }^{0} \mathbf{R}_{\mathrm{f}}{ }^{\mathrm{f}} \hat{\mathbf{u}},
$$

where ${ }^{0} \mathbf{R}_{\mathrm{f}}$ is the matrix rotation from the robot base frame to the initial probe frame $\mathcal{F}_{\mathrm{s}}(t=0)$. We finally obtain an estimation $\hat{\mathbf{L}}_{\mathbf{s}}$ of the interaction matrix by substituting in (17) the estimated unitary vectors ${ }^{\mathrm{S}} \hat{\mathbf{u}}_{1},{ }^{\mathrm{s}} \hat{\mathbf{u}}_{2}$ and the current coordinates $\mathbf{p}_{1}$ and $\mathbf{p}_{2}$ measured in the US image. An adaptive visual servoing is then performed by updating $\hat{\mathbf{L}}_{\mathbf{s}}$ at each iteration of the control law (20).

\section{RESULTS}

Here, we present simulation results of the adaptive control developed in Section 3. A software simulator was programmed in the MATLAB environment from the system modeling described in Section 2. The straight lines $\mathcal{D}_{1}$ and $\mathcal{D}_{2}$ are set with ${ }^{0} M_{1}={ }^{0} M_{2}=(0,0,0),{ }^{0} \mathbf{u}_{1}=(1,0,0)$ and ${ }^{0} \mathbf{u}_{2}=(0,1,0)$, and the initial position of the probe is fixed to ${ }^{0} \mathbf{t}_{\mathrm{s}}=(-0.12,-0.08,0.1)(\mathrm{m})$. To describe the rotation ${ }^{0} \mathbf{R}_{\mathrm{s}}$ we use the pitch-roll-yaw $(\alpha, \beta, \gamma)$ angles representation and the initial values are set to $\alpha \beta \gamma\left({ }^{0} \mathbf{R}_{\mathrm{s}}\right)=(-60,-160,90)(\mathrm{deg})$. The real spatial calibration parameters are set to ${ }^{{ }^{n}} \mathbf{t}_{\mathrm{s}}=(0.05,0,0), \alpha \beta \gamma\left({ }^{\mathrm{n}} \mathbf{R}_{\mathrm{s}}\right)=(0,0,0)$ and $S_{x}=S_{y}=0.0005$. The reference value of the visual features is set to the image center $\mathbf{s}^{*}=(0,0,0,0)$ and two references of the probe orientation are successively applied, the first $\alpha \beta \gamma\left({ }^{0} \mathbf{R}_{\mathrm{s}^{*}}\right)=(-40,-120,130)$ (deg) at the start and the second $\alpha \beta \gamma\left({ }^{0} \mathbf{R}_{\mathrm{s}^{*}}\right)=(-80,-100,45)(\mathrm{deg})$ at iteration $k=400$. The secondary task is considered in the control law only once the visual error norm of the first task is lower than 5 pixels in the image. The gain of the control law is fixed to $\lambda=0.8$ and 

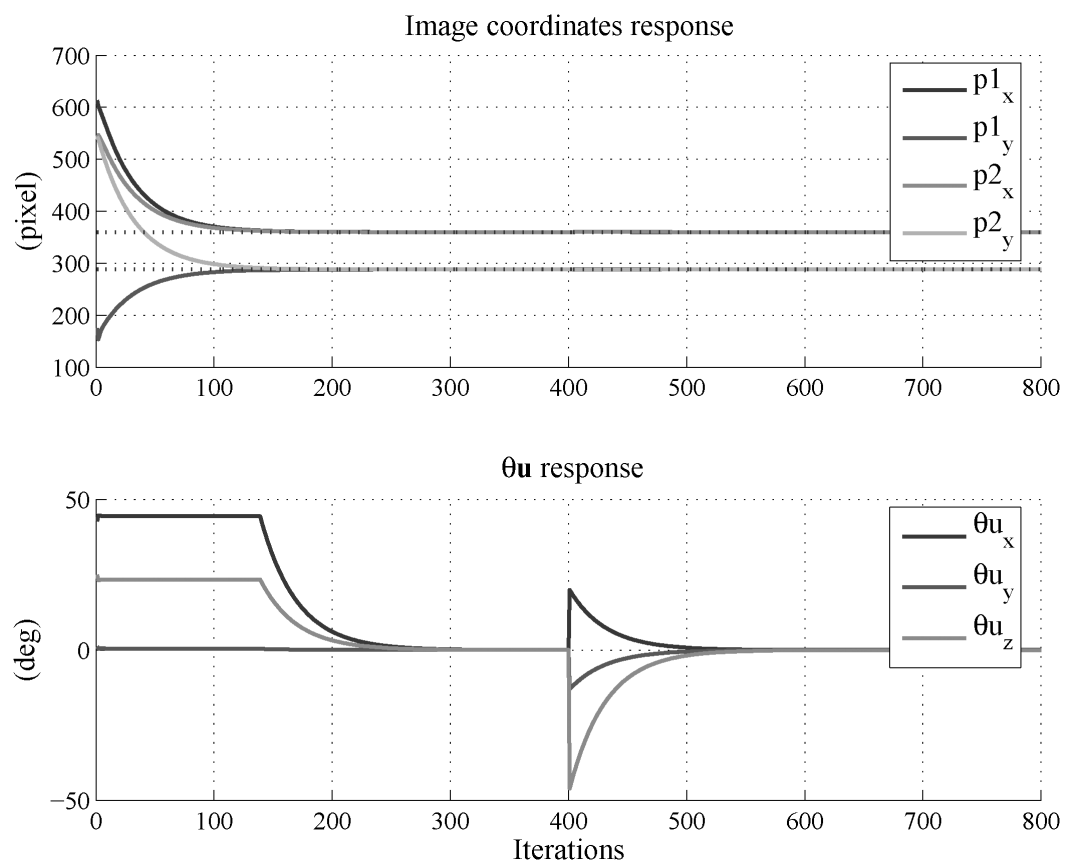

Figure 3. Image coordinates $\mathbf{p}_{1}$ and $\mathbf{p}_{2}$, and $\boldsymbol{\theta} \mathbf{u}$ error during the first simulation.

the initial estimated parameters of each straight lines are set to $\hat{a}=\hat{b}=\hat{c}=\hat{d}=1$. As we have noted in Section 3, the rank of the interaction matrix switches from 4 to 3 when points $P_{1} P_{2}$ join together. Of course joining the two points is the goal of the visual task, so we have to take into account the switching rank of the interaction matrix in the control law. When the two points join together (distance less than 5 pixels) we force the interaction matrix rank to 3 in order to avoid numerical instabilities.

In a first simulation we assume to know perfectly the spatial calibration parameters of the probe in order to show the ideal performance of the adaptive visual servoing. Figure 3 displays evolutions of image coordinates of the points and the $\boldsymbol{\theta} \mathbf{u}$ angle error of the secondary task. We can see that the two points converge exponentially towards the center of the image $(360,288)$ (pixels) and that the secondary task also decreases exponentially towards zero once the visual task is achieved without disturbing it. The image trajectories of the points are drawn in Fig. 4. We can note that at the start the points do not move towards the right direction because initial estimation values of ${ }^{\mathrm{s}} \mathbf{u}_{1}$ and ${ }^{\mathrm{s}} \mathbf{u}_{2}$ used to compute $\hat{\mathbf{L}}_{\mathbf{s}}$ are not accurate, but estimation values are quickly well readjusted by the on-line least-squares algorithm and then image trajectories become straight. Evolutions of the estimated unitary vectors of the straight lines are presented in Fig. 5. The bottom graphs correspond to the values ${ }^{0} \hat{\mathbf{u}}_{1}$ and ${ }^{0} \hat{\mathbf{u}}_{2}$ expressed in the robot base frame which is fixed with the scene and the top graphs to the values ${ }^{\mathrm{s}} \hat{\mathbf{u}}_{1}$ and ${ }^{\mathrm{S}} \hat{\mathbf{u}}_{2}$ obtained by projection into the probe frame. We can note that the values expressed in the robot base frame converge directly to 


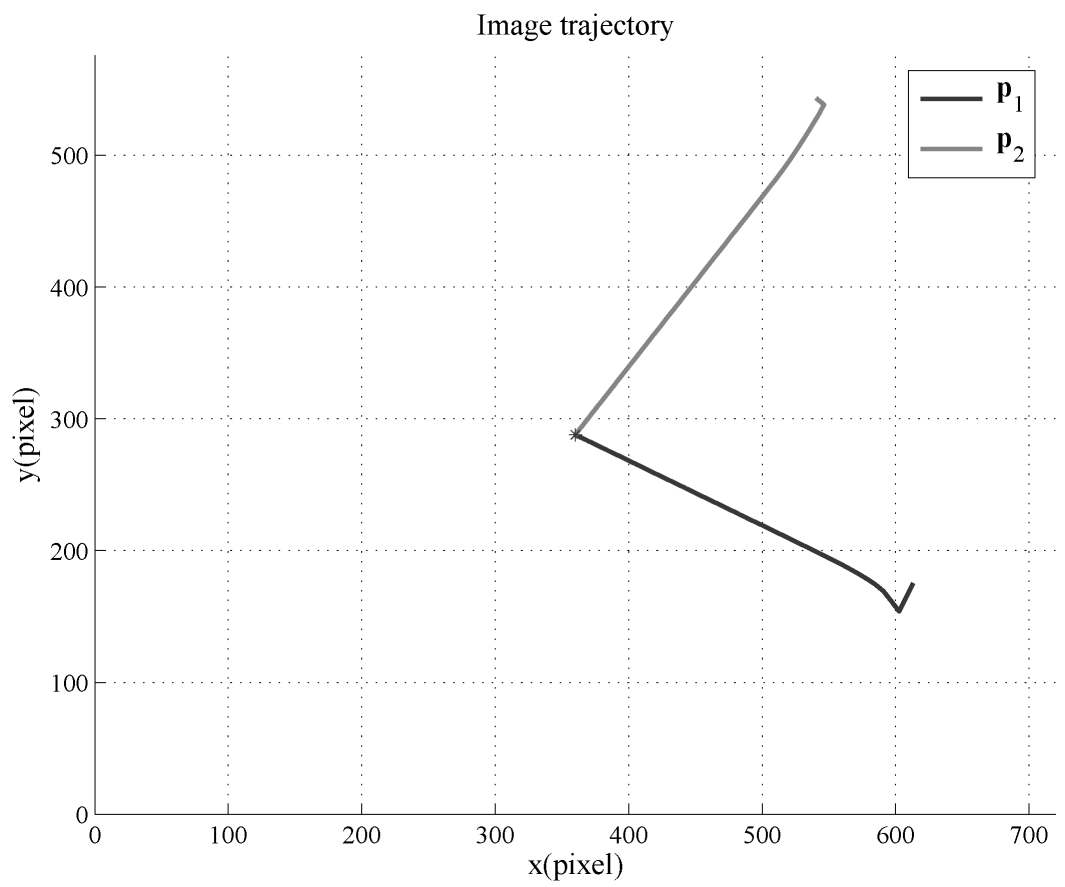

Figure 4. Image trajectories of points $P_{1}$ and $P_{2}$ during the first simulation.

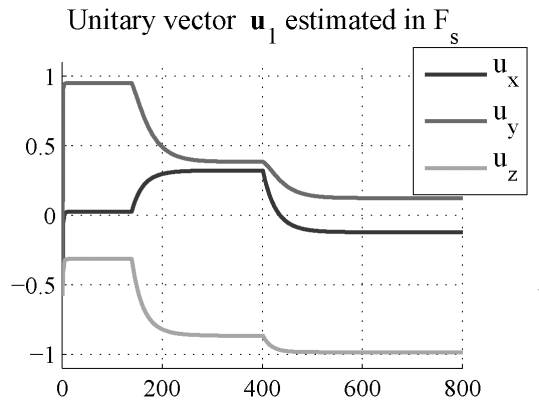

Unitary vectors $\mathbf{u}_{2}$ estimated in $\mathrm{F}_{\mathrm{s}}$

Unitary vector $\mathbf{u}_{1}$ estimated in $\mathrm{F}_{0}$
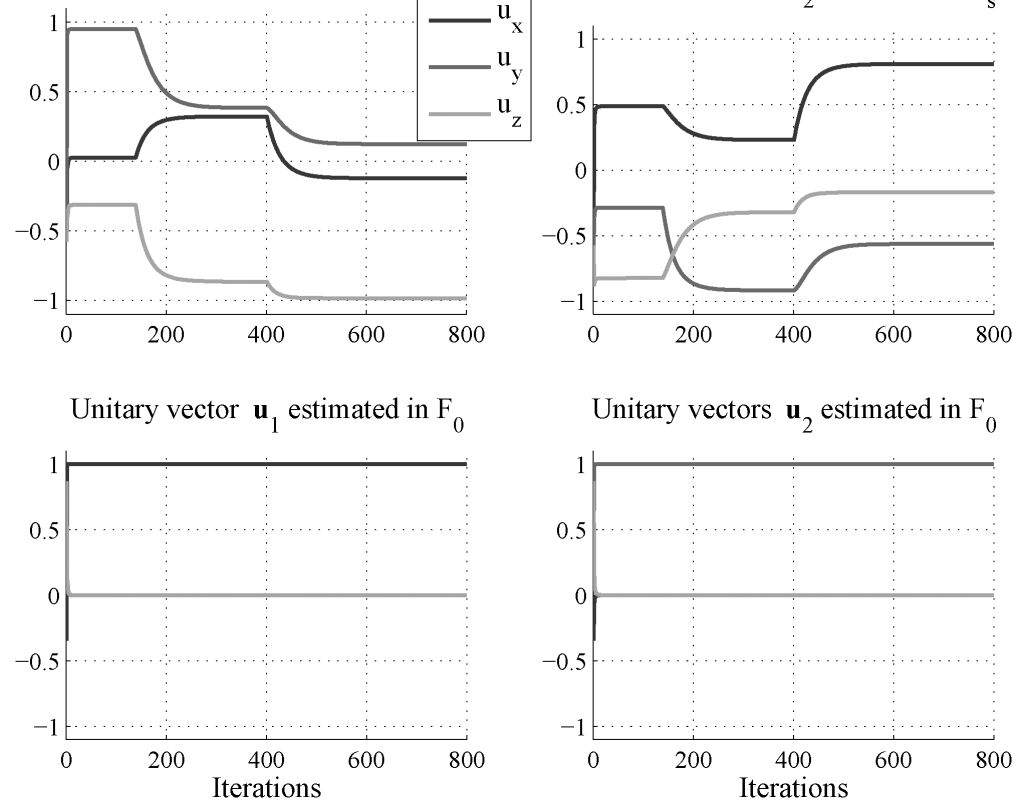

Unitary vectors $\mathbf{u}_{2}$ estimated in $\mathrm{F}_{0}$

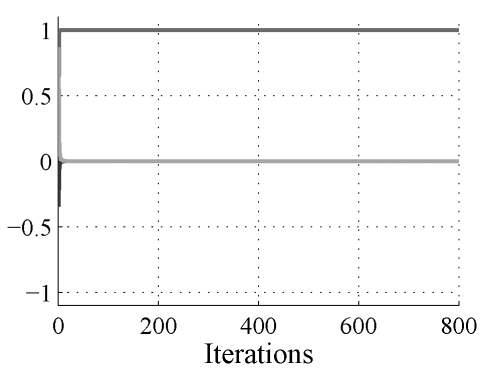

Figure 5. Estimation of unitary vectors $\mathbf{u}_{1}$ and $\mathbf{u}_{2}$ during the first simulation. 

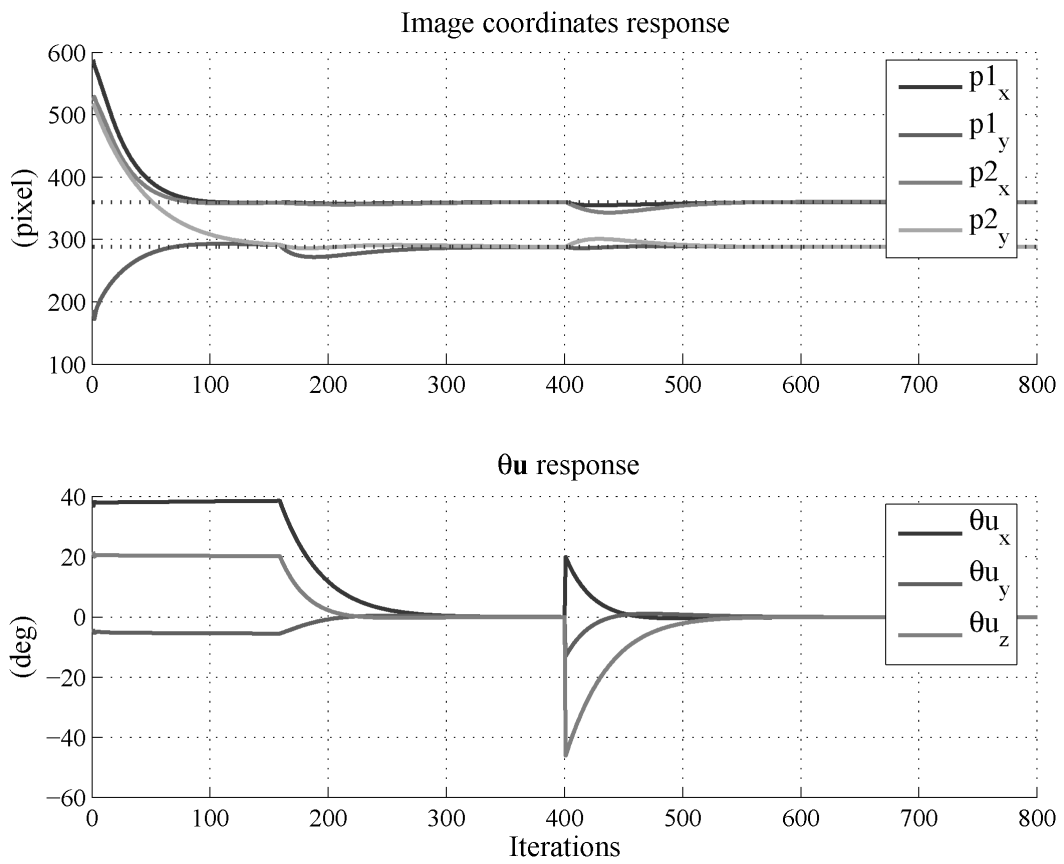

Figure 6. Image coordinates $\mathbf{p}_{1}$ and $\mathbf{p}_{2}$, and $\boldsymbol{\theta} \mathbf{u}$ error during the second simulation.

the real values ${ }^{0} \mathbf{u}_{1}=(1,0,0)$ and ${ }^{0} \mathbf{u}_{2}=(0,1,0)$ after two iterations. As a matter of course, the values expressed in the probe frame vary due to the displacement of the probe with respect to the scene.

In a second simulation we put significant error (about 10\%) on the spatial calibration parameters used by the control law and the least-squares algorithm. We set them to ${ }^{n} \mathbf{t}_{\mathrm{s}}=(0.045,-0.005,0.005)(\mathrm{m}), \alpha \beta \gamma\left({ }^{\mathrm{n}} \mathbf{R}_{\mathrm{s}}\right)=(5,5,5)$ (deg) and $S_{x}=S_{y}=0.00045$. Figures 6-8 present the same measurements as for the first simulation. However, we can see now that points trajectories in the image are little curved and that the visual task is lightly coupled with the secondary task. We can also see in Fig. 8 that estimation values of ${ }^{\mathrm{s}} \mathbf{u}_{1}$ and ${ }^{\mathrm{s}} \mathbf{u}_{2}$ expressed in the robot base frame are not exactly the same as the real one due to the model errors. Nevertheless, the robotic task is well performed due to the good robustness of the image-based visual servoing.

\section{CONCLUSION}

A new visual servoing technique based on 2-D US image has been presented to automatically position the US image plane on the intersection point of a crossed string phantom used for the spatial calibration of 3-D US system imaging. In our approach, we use the redundancy formalism to perform in the same time the visual task and a secondary task which consists in moving the probe to different 


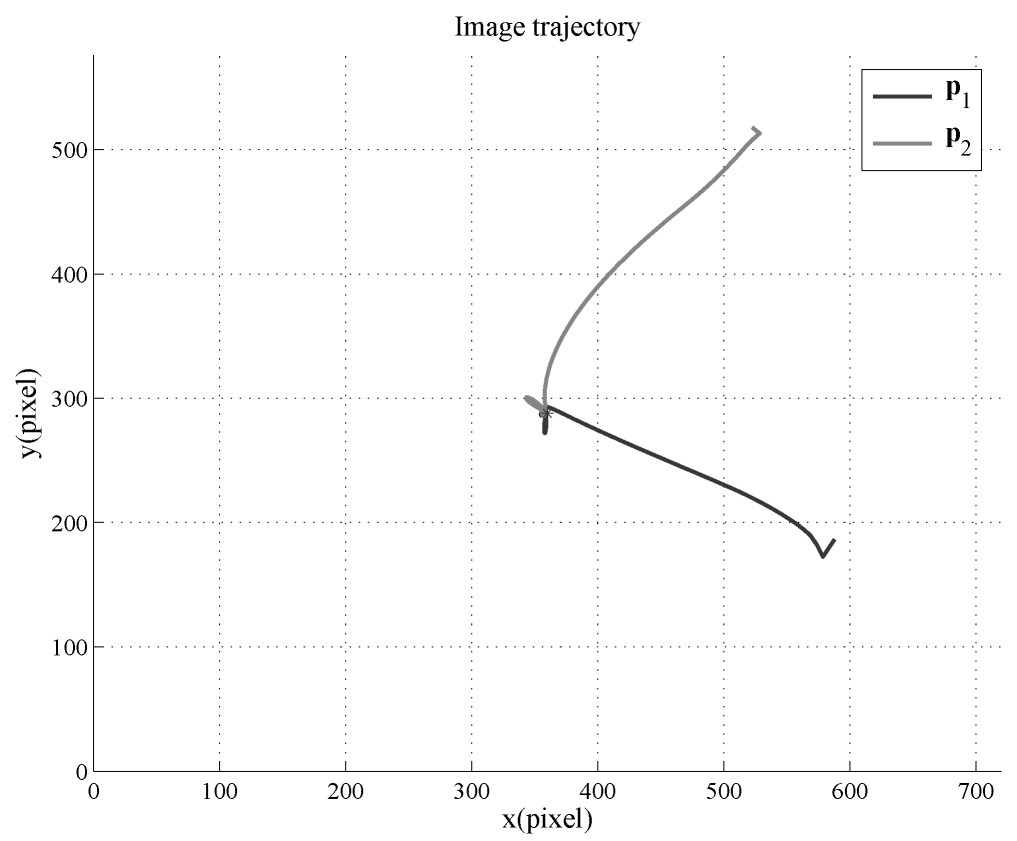

Figure 7. Image trajectories of points $P_{1}$ and $P_{2}$ during the second simulation.
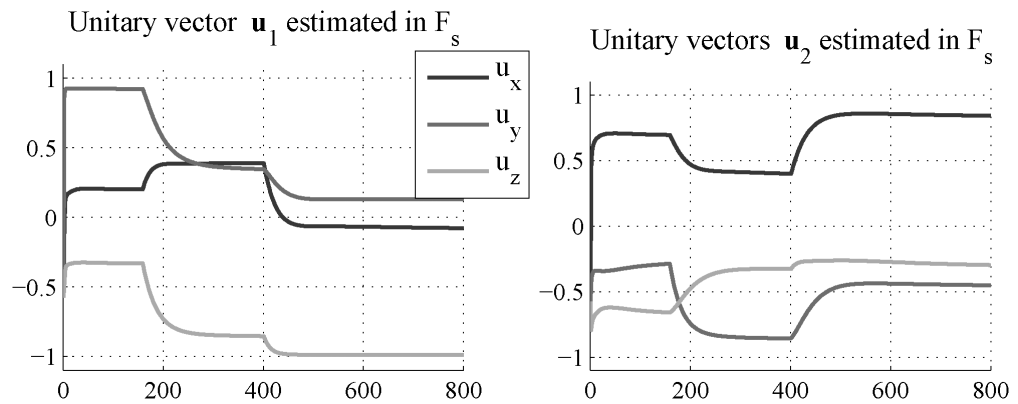

$$
\text { Unitary vector } \mathbf{u}_{1} \text { estimated in } \mathrm{F}_{0}
$$

$$
\text { Unitary vectors } \mathbf{u}_{2} \text { estimated in } \mathrm{F}_{0}
$$
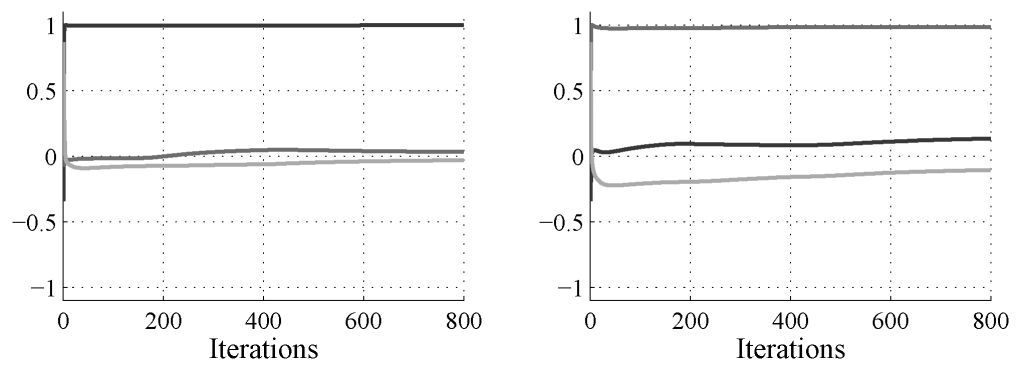

Figure 8. Estimation of unitary vectors $\mathbf{u}_{1}$ and $\mathbf{u}_{2}$ during the second simulation. 
orientations. An adaptive control law has been proposed by updating the interaction matrix related to the visual features thanks to an estimation algorithm. For the moment, results are obtained from simulation, but we plan to perform our task with a 6-d.o.f. medical manipulator specially designed for 3-D US imaging which will soon be available in our laboratory. Simulation results showed that the visual servoing is robust to large errors on the spatial calibration parameters.

\section{REFERENCES}

1. T. R. Nelson and D. H. Pretorius, Three-dimensional ultrasound imaging, Ultrasound Med. Biol. 24, 1243-1270 (1998).

2. P. R. Detmer, G. Basheim, T. Hodges, K. W. Beach, E. P. Filer, D. H. Burns and D. E. Strandness $\mathrm{Jr}$, 3D ultrasonic image feature localization based on magnetic scanhead tracking: in vitro calibration and validation, Ultrasound Med. Biol. 20, 923-936 (1994).

3. F. Rousseau, P. Hellier and C. Barillot, Robust and automatic calibration method for 3D freehand ultrasound, in: Proc. Int. Conf. on Medical Image Computing and Computer Assisted Intervention, Montreal, pp. 440-448 (2003).

4. K. Masuda, E. Kimura, N. Tateishi and K. Ishihara, Three-dimensional motion mechanism of ultrasound probe and its application for tele-echography system, in: Proc. IEEE/RSJ Int. Conf. on Intelligent Robots and Systems, Maui, HI, pp. 1112-1116 (2001).

5. A. Vilchis, J. Troccaz, P. Cinquin, K. Masuda and F. Pellisier, A new robot architecture for teleechography, IEEE Trans. Robotics Automat. 19, 922-926 (2003).

6. M. Mitsuishi, S. Warisawa, T. Tsuda, T. Higuchi, N. Koizumi, H. Hashizume and K. Fujiwara, Remote ultrasound diagnostic system, in: Proc. IEEE Int. Conf. on Robotics and Automation, Seoul, pp. 1567-1574 (2001).

7. F. Pierrot, E. Dombre, E. Degoulange, L. Urbain, P. Caron, S. Boudet, J. Gariepy and J. Megnien, Hippocrate: a safe robot arm for medical applications with force feedback, Med. Image Analysis 3, 285-300 (1999).

8. P. Abolmaesumi, S. E. Salcudean, W. H. Zhu, M. Sirouspour and S. P. DiMaio, Image-guided control of a robot for medical ultrasound, IEEE Trans. Robotics Automat. 18, 11-23 (2002).

9. J. Hong, T. Dohi, M. Hashizume, K. Konishi and N. Hata, An ultrasound-driven needle insertion robot for percutaneous cholecystostomy, Phys. Med. Biol. 49, 441-455 (2004).

10. M. A. Vitrani, G. Morel and T. Ortmaier, Automatic guidance of a surgical instrument with ultrasound based visual servoing, in: Proc. IEEE Int. Conf. on Robotics and Automation, Barcelona, pp. 510-515 (2005).

11. C. Samson, M. Le Borgne and B. Espiau, Robot Control: The Task Function Approach. Clarendon Press, Oxford (1991).

12. B. Espiau, F. Chaumette and P. Rives, A new approach to visual servoing in robotics, IEEE Trans. Robotics Automat. 8, 313-326 (1992).

13. E. Marchand and G.-D. Hager, Dynamic sensor planning in visual servoing, in: Proc. IEEE/RSJ Int. Conf. on Intelligent Robots and Systems, Leuven, Vol. 3, pp. 1988-1993 (1998).

14. G. D. Hager, Human-machine cooperative manipulation with vision-based motion constraints, presented at: Workshop on Visual Servoing (IROS'02), Lausanne (2002).

15. R. Johansson, System Modeling and Identification. Prentice-Hall, Englewood Cliffs, NJ (1993). 


\section{ABOUT THE AUTHORS}

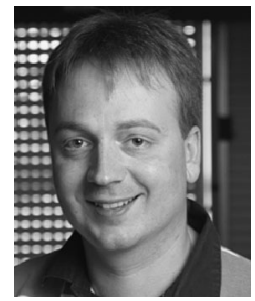

Alexandre Krupa received the MS and PhD degrees in Control Systems and Signal Processing from the National Polytechnic Institute of Lorraine, Nancy, France in 1999 and 2003, respectively. His PhD research work was carried out in the EAVR team with the Laboratoire des Sciences de l'Image de l'Informatique et de la Télédétectionthe, Strasbourg, France. From 2002 to 2004, he was Assistant Associate Professor for undergraduate student lectures in electronics, control and computer programming at Strasbourg I University. Since 2004, he has been a Research Scientist at the French National Institute for Research in Computer Science and Control and is member of the Lagadic group at IRISA/INRIA Rennes, France. His research interests include medical robotics, computer-assisted systems in the medical and surgical fields, and, most specifically, the control of medical robots by visual servoing and force control.

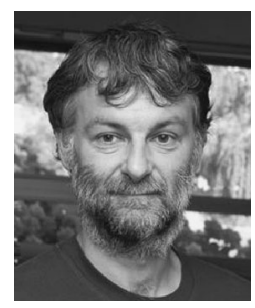

François Chaumette graduated from École Nationale Supérieure de Mécanique, Nantes, France, in 1987. He received the PhD degree in Computer Science from the University of Rennes in 1990. Since 1990, he has been with IRISA/INRIA, Rennes, France, where he is now Directeur de Recherches and Head of the Lagadic group. His research interests include robotics and computer vision, especially visual servoing and active perception. He received the AFCET/CNRS Prize for the best French thesis in automatic control in 1991. He also received with Ezio Malis the 2002 King-Sun Fu Memorial Best IEEE Transactions on Robotics and Automation Paper Award. He was Associate Editor of the IEEE Transactions on Robotics from 2001 to 2005 . 